\title{
EDITORIAL
}

\section{What is the role of microbial infection in Alzheimer's disease?}

\author{
Tatiana Barichello, ${ }^{1,2}$ iD Vijayasree V. Giridharan, ${ }^{1}$ (iD Clarissa M. Comim, ${ }^{3}$ Rodrigo Morales ${ }^{4,5}$ (iD) \\ ${ }^{1}$ Faillace Department of Psychiatry and Behavioral Sciences, McGovern Medical School, The University of Texas Health Science Center at \\ Houston (UTHealth), Houston, TX, USA. ${ }^{2}$ Laboratório de Fisiopatologia Experimental, Programa de Pós-Graduação em Ciências da Saúde, \\ Universidade do Extremo Sul Catarinense (UNESC), Criciúma, SC, Brazil. ${ }^{3}$ Laboratório de Neurociências, Universidade do Sul de Santa \\ Catarina (UNISUL), Tubarão, SC, Brazil. ${ }^{4}$ Department of Neurology, McGovern Medical School, UTHealth, Houston, TX, USA. ${ }^{5}$ Centro \\ Integrativo de Biología y Química Aplicada (CIBQA), Universidad Bernardo O'Higgins, Santiago, Chile.
}

In 1907, Alois Alzheimer, a German psychiatrist and neuropathologist, described the case of Auguste Deter, a 51-year-old woman who was closely monitored for 5 years for symptoms of progressive presenile dementia. After her death, necropsy revealed several abnormalities in her brain, including cortical atrophy, arteriosclerotic changes in vascular tissues, and the abnormal deposition of "special substances" inside and outside of neurons, now identified as hyperphosphorylated tau tangles and amyloid- $\beta(A \beta)$ plaques, respectively. ${ }^{1}$ Presently, Alzheimer's disease (AD) is considered the leading cause of dementia worldwide and is rapidly becoming one of the most costly, deadly, and burdensome diseases. ${ }^{2}$ Unfortunately, more than 100 years after its first description, we do not entirely understand how $A D$ is initiated and what factors modulate its progression. At present, we know that sporadic AD (accounting for over $95 \%$ of all $A D$ cases) can be linked with unmodifiable and modifiable risk factors. Aging is the most notorious risk factor for AD. Female sex and certain ethnicities have also been associated with a higher risk of developing this type of dementia. More recently, several pathological conditions such as type 2 diabetes, hypertension, and others have been linked with $A D$ at physiological and molecular levels. Among them, an emerging risk factor for $A D$ includes microbial infections. ${ }^{3}$ Basic and clinical data support the role of microbial infection in AD. Some of this evidence is discussed below.

One example found in the literature includes a multiscale analysis of three distinct patient cohorts demonstrating that human herpesvirus (HHV)-6A and HHV-7 disrupt molecular, genetic, and clinical networks more severely in $A D$ patients than in non-demented individuals. ${ }^{4}$ Another study showed that individuals recently diagnosed with herpes simplex virus (HSV, $\mathrm{n}=8,362$ ) had a risk ratio of 2.564 to develop dementia compared to non-HSV controls $(n=25,086)$. Notably, this risk ratio decreased to 0.092 when the HSV-infected patients received anti-herpetic treatment, ${ }^{5}$ suggesting

Correspondence: Tatiana Barichello, The University of Texas Health Science Center at Houston, 1941 East Road, Suite 3140, 77054, Houston, TX, USA.

E-mail: tatiana.barichello@uth.tmc.edu

Submitted Aug 25 2021, accepted Aug 25 2021, Epub Nov 012021. that the removal of pathogens results in an improved prognosis.

In the preclinical field, growing evidence suggests that infections might be associated with the accumulation of amyloid plaques in the brain, one of the hallmarks and perhaps the triggering event in $\mathrm{AD}$. One example includes experiments involving transgenic Caenorhabditis elegans expressing the human $A \beta 1-42$ residue. These animals, developing $A \beta$ deposits over time, displayed reduced mortality compared to their non-transgenic counterparts when infected with Candida albicans. ${ }^{6}$ Additional support comes from experiments involving the intracerebral injection of Salmonella Typhimurium in transgenic 5XFAD mice, an animal model of accelerated amyloid pathology. Treated mice exhibited increased brain amyloid pathology compared to sham-treated mice. Moreover, A $\beta$ deposits in Salmonella-treated 5XFAD co-localized with the invading bacteria, suggesting a direct interaction between both pathogenic entities (amyloid plaques and bacteria). Interestingly, the mortality rate induced by Salmonella $\mathrm{sp}$. administration was reduced in 5XFAD mice, consistent with previous in vitro and in vivo evidence suggesting an antimicrobial role for $A \beta$ aggregates. ${ }^{6}$ Corroborating the involvement of infection in $A D$ pathogenesis, we have demonstrated that pneumococcal meningitis increased the expression of the receptor for advanced glycation endproducts (RAGE), a known contributor for $A \beta$ production by enhancing the activity of secretases and neuroinflammation. We found that infection with Streptococcus pneumoniae also enhanced the generation of $A \beta 1-42$, an $A \beta$ fragment associated with increased fibrillogenesis, microglial cell activation, and memory impairment. ${ }^{7}$

Similar results were observed in models of peripheral infection triggered by polymicrobial sepsis using cecal ligation and puncture (CLP) surgery, the gold-standard model of polymicrobial sepsis. These animals displayed an increase in $A \beta$ production and higher Ser-202phosphorylated Tau ( $p$-TauSer-202, another indicator
How to cite this article: Barichello T, Giridharan VV, Comim CM, Morales R. What is the role of microbial infection in Alzheimer's disease? Braz J Psychiatry. 2022;44:245-247. http://dx.doi.org/ 10.1590/1516-4446-2021-0037 

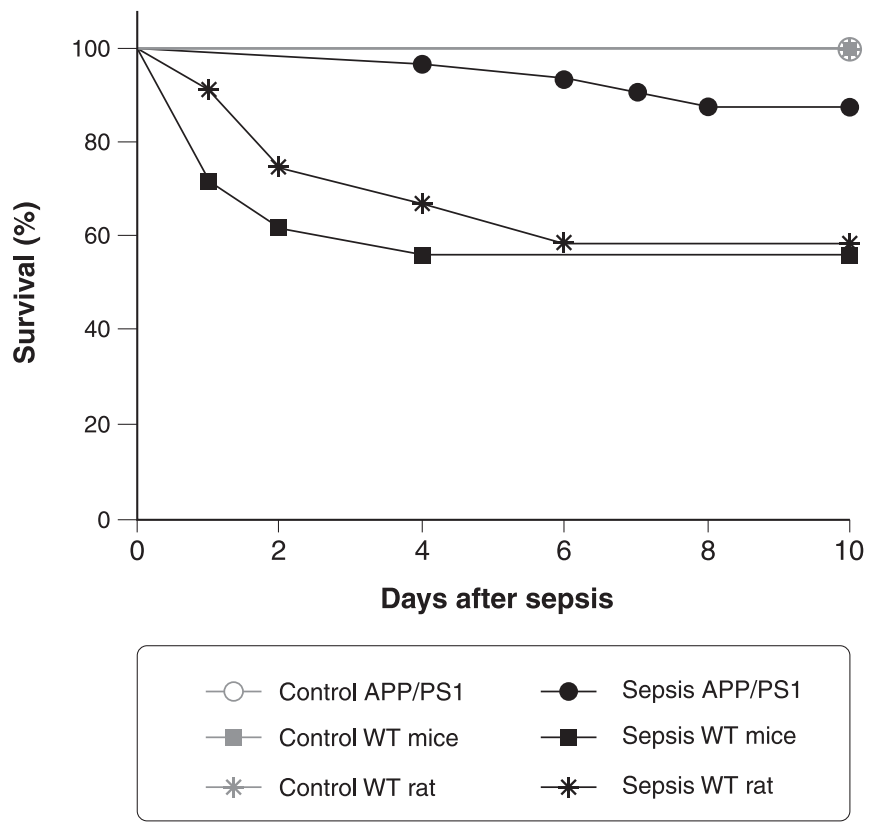

Figure 1 Kaplan-Meier survival curve of APP/PS1 mice, WT C57BL/6 mice, and Wistar rats subjected to experimental polymicrobial sepsis triggered by CLP. Control groups subjected to sham surgery were included for comparison. The animals were followed for 10 days and mortality was recorded (APP/PS1, sepsis $n=32$ vs. control $n=16, p=0.153$; C57BL/6 mice, sepsis $n=50$ vs. control $n=50, p<0.001$; Wistar rats, sepsis $n=12$ vs. control $n=10, p=0.02$ ). The APP/PS1 mice subjected to sepsis had an $87.88 \%$ survival rate, WT mice had a survival rate of $56 \%$, and Wistar rats had a survival rate of $58.33 \%$. APP/PS1 = amyloid- $\beta$ precursor protein/presenilin 1; CLP = cecal ligation and puncture.

of AD pathology) and RAGE followed by cognitive impairment. $^{8}$ In another study, APP/PS1 double transgenic mice that express human amyloid- $\beta$ precursor protein (APP) and a mutant human presenilin (PS1) associated with early-onset $A D$, were subjected to CLP and their survival was measured over time. Usually, CLP triggers sepsis of moderate severity, with mortality rate in rodents of about $40 \% .^{8,9}$ Some crucial variables that influence the mortality rate of the animals include fluid resuscitation procedures, CLP conditions, the position of the ligation, the postoperative antibiotic regimen, and parenteral nutrition. ${ }^{9}$ Surprisingly, we found that APP/ PS1 mice subjected to polymicrobial sepsis had a mortality rate of only $12.12 \%$, while the mortality of CLP in wild-type mice was $44 \%$ (Figure 1). This was also observed in a rat model of sepsis, where wild-type Wistar rats had a mortality rate of $41.6 \%$ compared with the respective controls. In summary, our data show that wild-type C57BL/6 mice and Wistar rats had a threefold increase in mortality compared to APP/PS1 mice. Our results are in agreement with those found in the literature, demonstrating that $A \beta$ provides protection during infection.
Neuroinflammation is a central component of AD pathogenesis; it contributes to disease development and neurodegeneration. ${ }^{2,3}$ Infection or inflammatory conditions derived from aging, as well as stroke, have been identified as AD risk factors, ${ }^{3}$ triggers of pro-inflammatory cytokine production in the brain, and drivers of neuroinflammation. Recently, an essential mechanism of cell signaling that associates neuroinflammation to $A \beta$ production was identified. In the brain, insults or infection lead to production of pro-inflammatory cytokines, which in turn promote the formation of interferon-induced transmembrane protein 3 (IFITM3) in neurons and astrocytes. IFITM3 binds to $\gamma$-secretase, increasing its activity, and upregulates $A \beta$ production. ${ }^{10}$ Figure 2 provides a summary of the molecular cascade associated with this pathway.

Overall, several mechanisms link microbial infections and $A D$, including but not limited to $A \beta$ misfolding and neuroinflammation. Additional research will help us clarify the specific molecular links between pathological events, explore the specificity of certain microbes with pathological variations in $A D$, and identify new preventive and therapeutic strategies to combat this fatal form of dementia. 


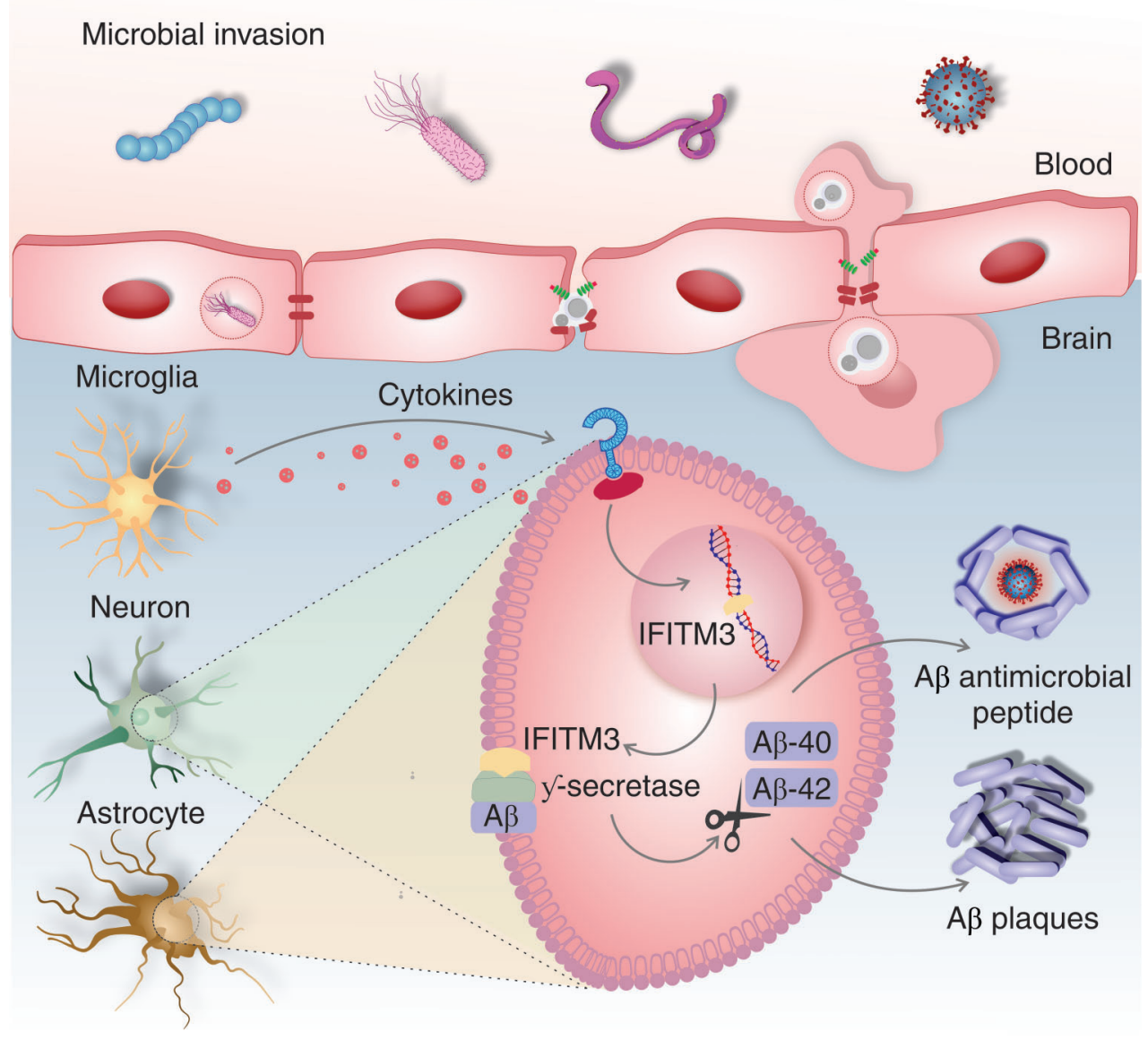

Figure 2 Infection-induced inflammation as a potential trigger of Alzheimer's disease pathology. Infection or inflammation activates microglial cells, triggering the release of pro-inflammatory cytokines. In sequence, the pro-inflammatory cytokines promote the formation of interferon-induced transmembrane protein 3 (IFITM3) in neurons and astrocytes, which binds to $\gamma$-secretase, increasing its activity and upregulating $A \beta$ production. Released $A \beta$ peptides have antimicrobial effects, preventing pathogen development by forming amyloid plaques. Brain $A \beta$ deposition exacerbates the cerebral inflammatory response, accelerating $A D$ pathological cascades. $A \beta=$ amyloid- $\beta ; A D=$ Alzheimer's disease.

\section{Acknowledgements}

This work was supported by The University of Texas Health Science Center at Houston. TB has received a grant from the Alzheimer's Association (AARGDNTF-19619645). TB and RM have received grants from the National Institutes of Health/National Institute on Aging (NIH/NIA grant 1RF1AG072491).

\section{Disclosure}

The authors report no conflicts of interest.

\section{References}

1 Alzheimer A, Stelzmann RA, Schnitzlein HN, Murtagh FR. An English translation of Alzheimer's 1907 paper, "Uber eine eigenartige Erkankung der Hirnrinde." Clin Anat. 1995;8:429-31.

2 Georges J, Miller O, Bintener C. Estimating the prevalence of dementia in Europe. Report N: ISBN 978-99959-995-9-9. Alzheimer Europe. 2020 Feb. DOI: 10.13140/RG.2.2.16880.81923.

3 Scheltens P, De Strooper B, Kivipelto M, Holstege H, Chételat G, Teunissen CE, et al. Alzheimer's disease. Lancet. 2021;397: 1577-90.
4 Readhead B, Haure-Mirande JV, Funk CC, Richards MA, Shannon P, Haroutunian V, et al. Multiscale analysis of independent Alzheimer's cohorts finds disruption of molecular, genetic, and clinical networks by human herpesvirus. Neuron. 2018;99:64-82.e7.

5 Tzeng NS, Chung CH, Lin FH, Chiang CP, Yeh CB, Huang SY, et al. Anti-herpetic medications and reduced risk of dementia in patients with herpes simplex virus infections-a nationwide, population-based cohort study in Taiwan. Neurotherapeutics. 2018;15:417-29.

6 Kumar DK, Choi SH, Washicosky KJ, Eimer WA, Tucker S, Ghofrani $\mathrm{J}$, et al. Amyloid- $\beta$ peptide protects against microbial infection in mouse and worm models of Alzheimer's disease. Sci Transl Med. 2016;8:340ra72.

7 Giridharan VV, Generoso JS, Collodel A, Dominguini D, Faller CJ, Tardin F, et al. Receptor for Advanced Glycation End Products (RAGE) mediates cognitive impairment triggered by pneumococcal meningitis. Neurotherapeutics. 2021;18:640-53.

8 Gasparotto J, Girardi CS, Somensi N, Ribeiro CT, Moreira JC, Michels $M$, et al. Receptor for advanced glycation end products mediates sepsis-triggered amyloid- $\beta$ accumulation, Tau phosphorylation, and cognitive impairment. J Biol Chem. 2018;293:226-44.

9 Rittirsch D, Huber-Lang MS, Flierl MA, Ward PA. Immunodesign of experimental sepsis by cecal ligation and puncture. Nat Protoc. 2009;4:31-6.

10 Hur JY, Frost GR, Wu X, Crump C, Pan SJ, Wong E, et al. The innate immunity protein IFITM3 modulates $\gamma$-secretase in Alzheimer's disease. Nature. 2020;586:735-40. 